\title{
Performance of the GRETA prototype detectors
}

\author{
K. Vetter, A. Kuhn, I.Y. Lee, R.M. Clark, M. Cromaz, \\ M.A. Deleplanque, R.M. Diamond, P. Fallon, G.J. Lane, \\ A.O. Macchiavelli, M.R. Maier, F.S. Stephens, C.E. Svensson, \\ H. Yaver \\ Lawrence Berkeley National Laboratory, Berkeley CA 94720, U.S.A.
}

\begin{abstract}
A working, two-dimensionally segmented Ge detector is one of the crucial elements in the development of GRETA - a next generation $4 \pi$ germanimum detector array that uses three-dimensional positions and energies to of individual interactions of $\gamma$ rays in the detector to reconstruct the full energies and direction vectors of the individual $\gamma$ rays by employing tracking algorithms. The threedimensional position and the energy of interactions will be determined by using a two-dimensionally segmented Ge detector along with pulse-shape analysis of the signals. The current prototype is a 36-fold segmented HP-Ge detector in a closed-ended coaxial geometry. Preamplifiers with a compact design, low noise, and very good response properties have been built and implemented. An integrated noise level of about $5 \mathrm{keV}$ has been measured for the segment channels. The average energy resolution of this detector was measured to be $1.14 \mathrm{keV}$ and $1.93 \mathrm{keV}$ at $60 \mathrm{keV}$ and $1332 \mathrm{keV}$, respectively. Using pulse-shape analysis, a three-dimensional position sensitivity of $0.2 \mathrm{~mm}$ to $0.5 \mathrm{~mm}$ ( $\mathrm{rms}$ ) has been obtained at $374 \mathrm{keV}$, dependent on the position and the direction. The results represent a major step towards the feasibility of a $\gamma$-ray tracking detector.
\end{abstract}

PACS: $07.85 ; 29.40 ; 29.40 . \mathrm{K} ; 29.40 . \mathrm{W}$

Key words: HPGe detectors; position-sensitive detectors; GRETA; gamma-ray tracking 


\section{Introduction}

Although Ge detectors have been used to detect $\gamma$ radiation for more than 30 years, new capabilities are still being developed. Constant improvements are being made in the manufacturing of these detectors, such as growing larger Ge crystals or lowering the impurity concentration to increase detection efficiency and peak-to-background ratio or to improve the energy resolution. Here, we report on another development of large coaxial HP-Ge detectors, namely the two-dimensional segmentation of the outer contact. It enables the determination of the three-dimensional position of $\gamma$-ray interactions by analyzing the shapes of the signals.

Segmentation of coaxial detectors in one dimension is being used already, e.g. in the Gammasphere detectors [1], primarily to reduce the Doppler broadening of $\gamma$ rays emitted in flight but also to measure the linear polarization of $\gamma$ rays [2]. Pulse-shape information has been used successfully in Ge detectors to improve energy resolution $[3,4]$, to perform Compton suppression $[5,6]$, or to determine the mean radius of interactions for an improved Doppler correction [7]. However, so far, in order to determine the location of interactions, neither a detailed analysis of the full signal as it arises during the charge collection nor the combination of segmentation and pulse-shape analysis have been performed.

The ability to determine the positions of $\gamma$-ray interactions in three dimensions is an important prerequisite for the GRETA project. GRETA, which stands for Gamma-Ray Energy Tracking Array, is currently being developed at the Lawrence Berkeley National Laboratory (LBNL) [8] and utilizes the new technique of $\gamma$-ray tracking. Tracking implies the determination of the time sequence of interactions of $\gamma$ rays in the detector and their respective positions and energies. Since the time resolution of Ge detectors is not sufficient to separate interactions in time, tracking algorithms based on positions and energies of individual interactions have to be used to determine the time sequence. Tracking not only increases the sensitivity for the detection of $\gamma$ radiation, improves background suppression and the capability to localize $\gamma$-ray sources, but also eliminates the need for Compton suppression used in the current generation of $\gamma$-detector arrays. Rather than suppress those $\gamma$ rays that escape from one Ge detector using a suppression shield (e.g. BGO), the goal is to use a shell of Ge detectors and to track the $\gamma$ ray throughout its path and recover its full energy. A much higher efficiency is achieved due to this add-back capability and the higher solid angle coverage of Ge detectors. Another advantage for using highly segmented Ge detectors is the higher overall count rate capability due to the large number of segments.

While GRETA focuses on improving the performance of $\gamma$-ray spectrometers primarily in the field of nuclear structure physics, the technique of $\gamma$-ray tracking will also allow improvements in other areas, such as in astrophysics or medical $\gamma$-ray imaging. 
Research and development for GRETA have focussed on three areas: 1.) The manufacture of a highly segmented coaxial Ge detector with low noise and fast preamplifiers; 2.) Determination of position sensitivity in three dimensions and development of algorithms to decompose signals from multiple interactions to determine their positions and energies; 3.) Development of $\gamma$-ray tracking algorithms for resolving tracks of multiple and coincident $\gamma$ rays. The subject of this paper is the first aspect and focuses on test results of a 36-fold segmented Ge detector. We will also briefly discuss results from the first prototype tested, which was a 12-fold segmented Ge detector. Results of position sensitivity measurements are presented in detail in a separate paper [9] and the results of a tracking algorithm developed in connection with the GRETA project are discussed in reference [10].

\section{The GRETA detector}

The ability to manufacture coaxial Ge detectors with a high degree of twodimensional segmentation is an essential component of our approach towards $\gamma$-ray tracking. The combination of segmentation and pulse-shape processing of segment signals provides the energies and positions of interaction points which are used as input for tracking algorithms to identify and separate individual $\gamma$ rays and to determine the time sequence of the interactions.

Since GRETA focuses on the implementation of $\gamma$-ray tracking for a $4 \pi \gamma$ ray detector array, many of these Ge detectors have to be closely packed to maximize the solid angle coverage. Several packaging schemes are possible, such as a spherical shell, or barrel-, or cube-like arrangements. The prototype detectors we obtained are designed to fit into a spherical shell of about 100 tapered hexagonal and pentagonal detectors, very similar to the Gammasphere geometry [1]. The 36-fold segmented prototype and its segmentation scheme is shown in fig. 1. It consists of a closed-ended HP-Ge n-type crystal with a tapered hexagonal shape. The length of the crystal is $9 \mathrm{~cm}$, the diameter at the back is $7 \mathrm{~cm}$ and the maximum diameter at the front is $4.4 \mathrm{~cm}$. The inner hole has a length of $7.5 \mathrm{~cm}$ and a diameter of $1.0 \mathrm{~cm}$. The angle of the taper is 10 degrees. The outer electrode is divided into 36 parts by 6 longitudinal and 5 transverse segmentation lines. The 6 longitudinal boundaries are located in the middle of the flat surfaces of the hexagonal shape. The widths of the transverse segments starting at the front (the narrow end) are $7.5 \mathrm{~mm}$, $7.5 \mathrm{~mm}, 15 \mathrm{~mm}, 20 \mathrm{~mm}, 25 \mathrm{~mm}$ and $15 \mathrm{~mm}$, respectively. The thicknesses of the layers were chosen to distribute the number of the interactions more equally among the segments for $\gamma$ rays coming from the front and to allow the study of the effects of different thicknesses on the transient-signal sensitivity. The Ge crystal resides in a $1 \mathrm{~mm}$ thick aluminum can of the same shape as the crystal. This can is separated from the crystal by $1 \mathrm{~mm}$ to simulate the 
close packed geometry of individually encapsulated detectors, similar to the design of the Cluster detectors [11] currently implemented in the Euroball array. The first prototype detector tested consisted of the same crystal, housing and cryostat but had only 12 segments, 6 longitudinal and 2 transverse. Both detectors were built by Eurisys Mesures in Strasbourg. The 13 (12 segments +1 central channel) FET's of the first and the 37 FET's (36 segments +1 central contact) of the second prototype are located and cooled in the same vacuum as the crystal. Cold FET's provide low noise, which is important for optimizing the energy and position resolution.

\section{The preamplifiers}

Although current, commercially available preamplifiers are able to provide very good energy resolution values, they do not meet our requirements of compact size, fast rise times or resistence to ringing. Therefore, new preamplifiers were designed and built at LBNL and mounted on the 36-fold segmented prototype detector. They were built using the latest small (surface mount) components and designed to have low power consumption, fast rise time, and a frequency-independent input impedance. They are characterized by their small size, low noise, and excellent response properties [12]. It is possible to mount all 37 preamplifiers radially on a cylindrical motherboard close to the feed-throughs on the back of the detector. This geometry minimizes external wiring which reduces inductance on the inputs of the preamplifiers. It furthermore avoids crossing of wires from different segments which could contribute to cross-talk. Special care was taken in filtering of the power supplies. With a pulser, an energy resolution of about $900 \mathrm{eV}$ was obtained for the preamplifiers, which is dominated by the FET noise. No overshoot is observed down to rise times as low as 10 ns. This was measured with an input capacitance of $10 \mathrm{pF}$ and translates into a bandwidth of $35 \mathrm{MHz}$. Each preamplifier is connected by pins to sockets on the motherboard, which allows easy mounting and dismounting.

\section{Performance of the prototypes}

In this section we show results of measurements on energy resolution, noise properties, three-dimensional position sensitivity, and the relative efficiencies of individual segments. In addition, we discuss measured cross-talk and its interpretation. 


\subsection{Energy resolution}

The energy resolution is one important characteristic in the performance of a $\gamma$-ray detector. It reflects the quality of the crystal and charge collection properties, contributions from the parallel noise, e.g. given by the leakage current or transistor base current, as well as series noise contributions, e.g. given by the transconductance of the FET and the detector capacitance $[13,14]$. While the energy resolution at low energies (e.g. $60 \mathrm{keV}$ from a ${ }^{241} \mathrm{Am}$ source) characterizes the noise contribution, the resolution at a higher energy (e.g. $1332 \mathrm{keV}$ from a ${ }^{60}$ Co source) reflects the charge collection properties.

Fig. 2 shows energy resolution values measured for the first, 12-fold segmented detector, by the manufacturer and in our laboratory for energies of $60 \mathrm{keV}$ and $1332 \mathrm{keV}$. The average resolution values for all functional segments measured at LBNL are $1.10 \mathrm{keV}$ and $1.90 \mathrm{keV}$, respectively. The central channel was measured to be $1.08 \mathrm{keV}$ and $2.05 \mathrm{keV}$. All energy resolution measurements were performed with a $3 \mu$ s shaping time. While the central channel resolution is comparable with good n-type Ge detectors, the segment channel resolution is better than the central channel and far superior to the segment resolution of the two-fold segmented Gammasphere detectors of about $5 \mathrm{keV}$. This is predominantly due to the small segment size and therefore smaller capacitance which decreases the series noise contribution.

In fig. 3 values of energy resolution of the second, 36-fold segmented detector, are shown for the same energies. As mentioned earlier, the second prototype consists of the same crystal and cryostat as the first prototype, only the number of segments and therefore the number of FET's in the vacuum was increased. Due to the large heat dissipation of the 37 FET's of about $2 \mathrm{~W}$, the cryostat is not able to provide sufficient cooling power if all channels are switched on. The characteristic feature of this effect is a degradation of the energy resolution as a function of time which reflects the warming up of the FET's until a thermal equilibrium is achieved after about 3 hours and the energy resolution stays constant. No shift of the baseline can be observed indicating that only the FET's are warming up and not the crystal which can be explained by the small thermal coupling between the board containing the FET's and the crystal. This reduced thermal contact keeps the crystal at lower temperatures than the FET's. Furthermore, a temperature sensitive resistor (PT100), which is located close to the Ge crystal, indicated only a slight temperature increase of $2 \mathrm{~K}$. The particularly bad resolution values for segment D5 in fig. 3 can be explained by the fact that segments D5 and D6 are shorted resulting in a bigger segment with larger capacitance. The open points in fig. 3 indicate the energy resolution values which have been obtained after all channels were powered for one day. Although the FET's have not been at an optimum temperature, average values of $1.41 \mathrm{keV}$ and $2.15 \mathrm{keV}$ at an energy of $60 \mathrm{keV}$ and $1332 \mathrm{keV}$ were measured, respectively. When measurements were performed immediately after powering FET's and preamplifiers 
or with only half of the channels switched on, average values of $1.14 \mathrm{keV}$ and $1.93 \mathrm{keV}$ with a spread of $0.08 \mathrm{keV}$ ( $\mathrm{rms}$ ) could be obtained. These excellent energy resolution values are very similar to the values obtained with the first prototype. The problems associated with the insufficient cooling power can be solved by redesigning the cryostat.

\subsection{Noise properties}

The study of the noise response is useful to determine further properties of the detection system. We will discuss the noise in terms of the integrated power spectrum as a function of frequency. An example of a noise spectrum is plotted in the upper part of fig. 4. It shows the measured charge amplitude in $\mathrm{keV}$ as a function of time. This example was obtained by digitizing the charge signal of a segment with a waveform digitizer at a sampling rate of $500 \mathrm{MHz}$ and an amplitude resolution of 8 bits. To match the input range of the waveform digitizers of $250 \mathrm{mV}$, the signals were amplified by a factor of $100 \mathrm{using}$ the Philips amplifier model 776 twice. The amplifiers have a bandwidth of $200 \mathrm{MHz}$, which is sufficient for this measurement. This amplification resulted in a gain of about $6 \mathrm{~V} / \mathrm{MeV}$ which for the observed noise level of about $5 \mathrm{keV}$ meant a charge amplitude of about $30 \mathrm{mV}$, clearly larger than the digitizer noise.

While the middle part of fig. 4 shows the power spectrum of the noise spectrum above, the bottom part displays the integrated power spectrum. The linear increase in the integrated power spectrum up to $3.5 \mathrm{keV}$ at $36 \mathrm{MHz}$ reflects a white noise contribution and the slope is predominantly given by FET properties and the detector capacitance. This frequency range implies a simple response of the system which is particularly useful for pulse-shape analysis. For frequencies higher than $40 \mathrm{MHz}$ the increase of the integrated power becomes smaller and stays almost constant at about $4.5 \mathrm{keV}$ beyond $60 \mathrm{MHz}$. This observed noise response is typical for all segment channels. The low value of $3.5 \mathrm{keV}$ at the maximum frequency of the linear response or $4.5 \mathrm{keV}$ of the total integrated noise are important factors for the position resolution and will be discussed in the following section. It also determines the low energy threshold for the identification of an energy deposition in a segment.

\subsection{Position resolution}

Besides the ability to manufacture a highly segmented coaxial Ge detector with excellent energy resolution and good noise response properties, the position resolution achievable in three dimensions is another important characteristic in the performance of a tracking detector. 
Using both the signal from the charge collecting electrode and the transientimage charge of its neighbors allows us to determine the positions of $\gamma$-ray interactions with a higher resolution than the size of the electrodes. While it is well known that the radial component of the interaction is implicitly contained in the drift time of the charge carriers [15], the two other dimensions can be obtained by a two-dimensional segmentation of one of the electrodes, in our case the outside boron contact. It has been demonstrated that a position sensitivity of $0.2 \mathrm{~mm}-0.5 \mathrm{~mm}$ (rms), depending on the position and direction, can be achieved for an energy of $374 \mathrm{keV}$ [9]. For an energy of $100 \mathrm{keV}$, a sensitivity of about $1 \mathrm{~mm}$ can still be expected. These excellent values have been obtained in a collimated coincidence set-up which determined the location of single interactions in the volume of the crystal. Sensitivity as it is defined in [9] and used in the following measures the minimum distance between interactions that produce distinguishable signals. A high sensitivity refers to a small distance.

To clarify the following discussion, the lay-out with the numbering of the segments is shown in fig. 5. In total, signals from 36 different positions of interaction points in segment B4 have been measured. These points are arranged in 3 layers in the depth, each with the 12 plotted positions as indicated in fig. 5. One example of a set of measured signals is shown in fig. 6. For comparison, calculated signals based on the same location of the interaction are shown as dashed lines. While B4 contains the interaction which is close to the front neighbor segment B3 and azimuthal neighbor C4 and exhibits a net charge signal, the neighbors only show a transient charge signal. It has been shown that the spread of the different signals shown in fig. 6 is mainly due to the finite opening angle of the collimation system which restricts the volume of possible interactions to a cylinder of about $1.5 \mathrm{~mm}$ diameter and $1.9 \mathrm{~mm}$ depth. Fig. 7 shows sensitivities obtained for a location indicated by the arrows relative to the other 35 positions which have been measured. It quanitfies how well one can distinguish between signals of the given position of $\mathrm{x}=22 \mathrm{~mm}$, $\mathrm{y}=1.5 \mathrm{~mm}$ and $\mathrm{z}=55.5 \mathrm{~mm}(\Delta \mathrm{z}=4.5 \mathrm{~mm})$ and all other measured positions. Each of the two-dimensional spectra represents all 12 positions in one depth (z) layer. Analyzing not only adjacent but all measured positions ensures that the obtained set of signals is unique. The upper part shows the measured and the bottom part the calculated sensitivities. First of all, a good agreement can be obtained between the measurements and the calculations. With this plot the sensitivities for different direction can be easily seen. While the sensitivity along $\mathrm{x}$, which is similar to the radius, is about $0.2 \mathrm{~mm}$, the sensitivity along $\mathrm{y}$ and $\mathrm{z}$, which are directions perpendicular to the segmentation lines, are on the order of $0.5 \mathrm{~mm}$. The difference can be understood in terms of a larger variation in the shape for changing radii than for the other directions. Since the underlying mechanism of the transient induced signal along the $\mathrm{y}$ and $\mathrm{z}$ direction is the same, the sensitivity along these directions is comparable. Fig. 7 only shows one example out of 36 positions analyzed. However, it represents a typical case. The distribution of all measured and calculated position sensitiv- 
ities are shown in fig. 8. The average values are $0.36 \mathrm{~mm}$ and $0.33 \mathrm{~mm}$ with a width of $0.13 \mathrm{~mm}$ and $0.18 \mathrm{~mm}$ for the measured and simulated distributions, respectively.

We have to emphasize that we use the word position sensitivity rather than position resolution for several reasons: position resolution implies that it is possible to absolutely locate one or several interactions of a $\gamma$ ray in the Ge crystal. In contrast, a position sensitivity only quantifies the relative difference of signals for different positions. Important effects not included in the determination of the position sensitivity are the finite range of the Compton electron in Ge, and the difficulty of determining the absolute position of the crystal. In fact, the range of the Compton electron will limit the position resolution to about $1 \mathrm{~mm}$ (e.g. for a 90 degree Compton scattering of a $1 \mathrm{MeV} \gamma$ ray), but nonetheless still satisfies the requirement for efficient tracking.

\subsection{Relative efficiencies of the segments}

Another characteristic of a segmented Ge detector is the relative efficiency of the individual segments. It reflects the charge collection properties for a specific volume in the detector, which depends on the crystal properties and the geometry of the crystal boundaries and segmentation lines. Fig. 9 shows measured and calculated relative efficiencies for the B segments as a function of depth. The figure shows values obtained for a $\gamma$-ray energy of $662 \mathrm{keV}$ (top) and $1332 \mathrm{keV}$ (bottom). The solid line shows the measured intensities. The dotted line shows intensities obtained by simulation calculations only based on the locations where the interactions occurred, assuming the charge would be collected in the segment in which the interaction took place. More realistic calculations based on this Monte-Carlo simulations but also taking into account the pathway of the charge carrier and where they are eventually collected are shown as dashed lines. Clearly, to reproduce the intensity profile, the electrical field has to be taken into account. The segment dimensions are not simply related to its efficiency. This can be easily seen in the segments of the first and second layer from the front of the crystal, which have approximately the same size. Due to the closed-ended and tapered geometry, many more field lines end on the front segment compared to the adjacent segment in the depth. A good agreement between measurements and calculations, in particular of the shape of the distributions, can be observed, indicating that the segments perform as expected. The geometry of the electrical field and its impact on the charge carrier pathways has to be taken into account in the design of segmented detectors, e.g. when the goal of the segmentation geometry is to distribute interactions of $\gamma$ rays equally over all segments. 


\subsection{Cross-talk}

Due the relatively high density of electronics in the 36-fold segmented GRETA prototype, shielding of the individual channels against cross-talk is crucial. This applies to the FET's, the wiring, the feed-throughs and the preamplifiers. Although care was taken to minimize the cross-talk, we observe a coupling between some of the segments. It occurs only between adjacent channels on the FET board. The main characteristic of this cross-talk is a net charge in both adjacent channels with opposite polarity of the original net charge signal. This cross-talk can be compensated by adding a fraction of about $3 \%$ of the original net charge signal. It can be explained by an insufficient decoupling of the power supply for the FET's. Another type of cross-talk is observed only to one of the neighboring segments. This cross-talk is based on

an inductive coupling which can be compensated by subtracting a fraction of the derivative of the original net charge signal. This cross-talk indicates an insufficient shielding between either the input or the output of the FET's and the asymmetric arrangement of wires of one adjacent segment. In fig. 6 both types of cross-talk were corrected in software. However, in fig. 10 signals in segments B3 and B5 are shown before and after the correction. In summary, the origin of the cross-talk is understood and can be corrected. Since the crosstalk is due to insufficient shielding, a better electronics lay-out should be able to eliminate it.

\section{Conclusions}

Two-dimensionally segmented, coaxial HP-Ge detectors have been studied. Properties of the prototype detectors have been measured in terms of energy resolution, noise response, and three-dimensional position sensitivity. Excellent energy resolution values of about $1.1 \mathrm{keV}$ and $1.9 \mathrm{keV}$ on average, for $60 \mathrm{keV}$ and $1332 \mathrm{keV}$, respectively, have been obtained. A linear dependence has been found for the integrated power spectrum of the noise as a function of frequency up to about $35 \mathrm{MHz}$. At this frequency the noise amplitude is integrated to about $3.5 \mathrm{keV}$ while the maximum integrated noise at higher frequencies was determined to be in the order of $5 \mathrm{keV}$. These low values and the very good step response characteristic of the detection system is of significant importance for the measured three-dimensional position sensitivity of $0.2 \mathrm{~mm}$ to $0.5 \mathrm{~mm}$ at an energy of $374 \mathrm{keV}$.

These results indicate that this detector can satisfy the requirements for $\gamma$-ray tracking and constitutes a major step in realizing the GRETA concept. 


\section{Acknowledgements}

We would like to thank Eurisys Mesures for providing the prototype detectors. We also thank Jerry Blair and Bill Payne of Bechtel for supplying the data acquisition system and Greg Schmid and Dean Beckedahl of LLNL for many stimulating discussions. This work was supported by the Director, Office of

Energy Research, Division of Nuclear Physics of the Office of High Energy and Nuclear Physics of the U.S. Department of Energy under contract No. DE-AC03-76SF0093. 


\section{References}

[1] I Y. Lee, Nucl. Phys. A 520 (1990) 641c

[2] G.J. Schmid, et al., Nucl. Instr. and Meth. A 417 (1999) 95

[3] W. Gast, et al., Nucl. Instr. and Meth. A 171 (1980) 49

[4] B. Hubbard-Nelson, et al., Nucl. Instr. and Meth. A 422 (1999) 411

[5] G.J. Schmid, et al., Nucl. Instr. and Meth. A 422 (1999) 368

[6] B. Philour, et al., Nucl. Instr. and Meth. A 403 (1998) 136

[7] Th. Kröll, et al., Nucl. Instr. and Meth. A 371 (1996) 489

[8] M.A. Deleplanque, et al., Nucl. Instr. and Meth. A 430 (1999) 292

[9] K. Vetter, et al., submitted to Nucl. Instr. and Meth. A

[10] G.J. Schmid, et al., Nucl. Instr. and Meth. A 430 (1999) 69

[11] J. Eberth, et al. Nucl. Instr. and Meth. A 369 (1996) 135

[12] M.R. Maier, et al., submitted to the Proceedings of the IEEE Symposium on Nuclear Science, Seattle Oct. 1999

[13] F.S. Goulding, et al., IEEE NS-29,3 (1982) 1125

[14] V. Radeka, Ann. Rev. Nucl. Part. Sci. 38 (1988) 217

[15] J. Blair, et al., Nucl. Instr. and Meth. A 422 (1999) 331 


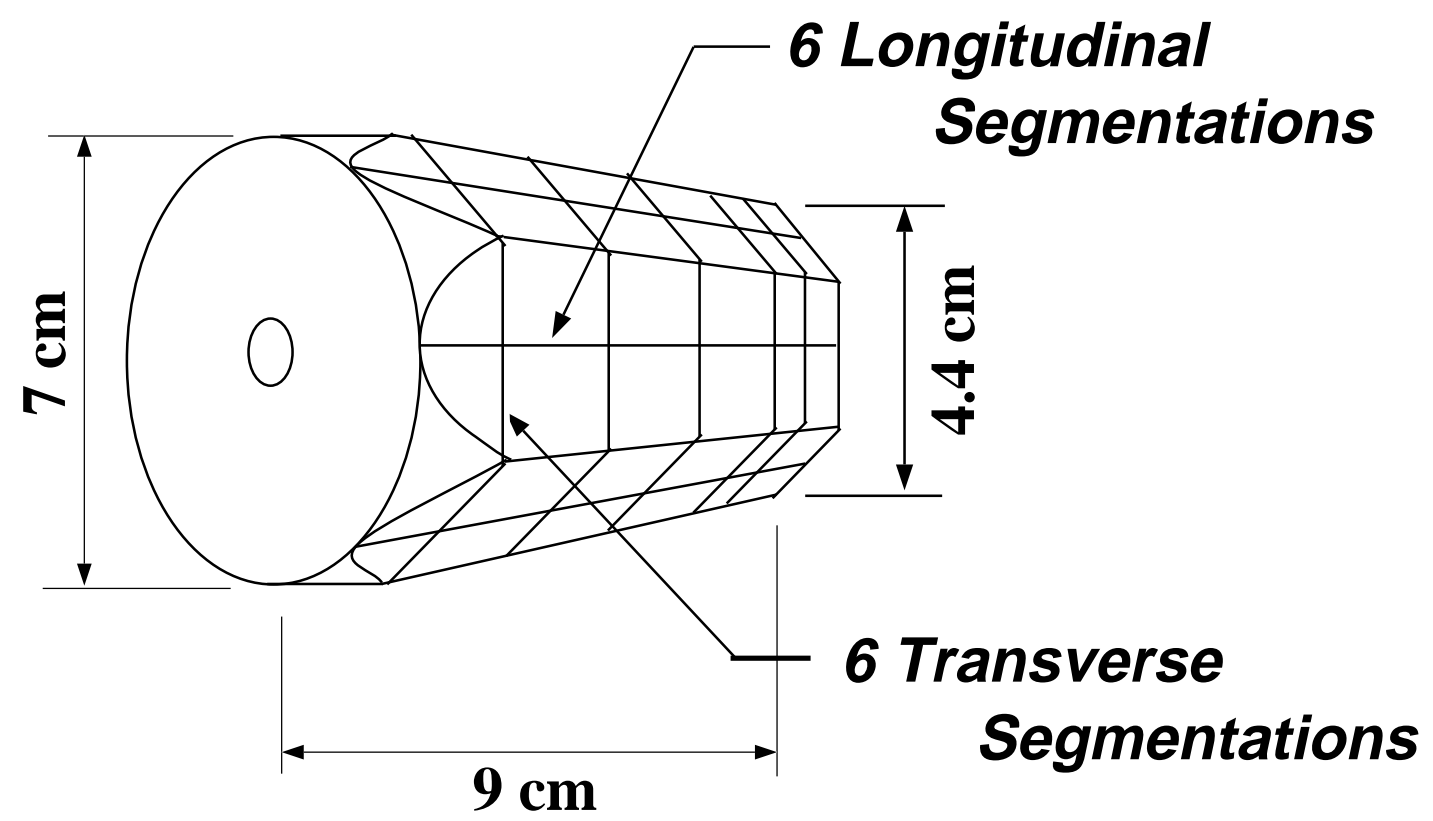

Fig. 1. The 36-fold segmented GRETA prototype detector with its tapered and hexagonal shape and the arrangement of its segments. 


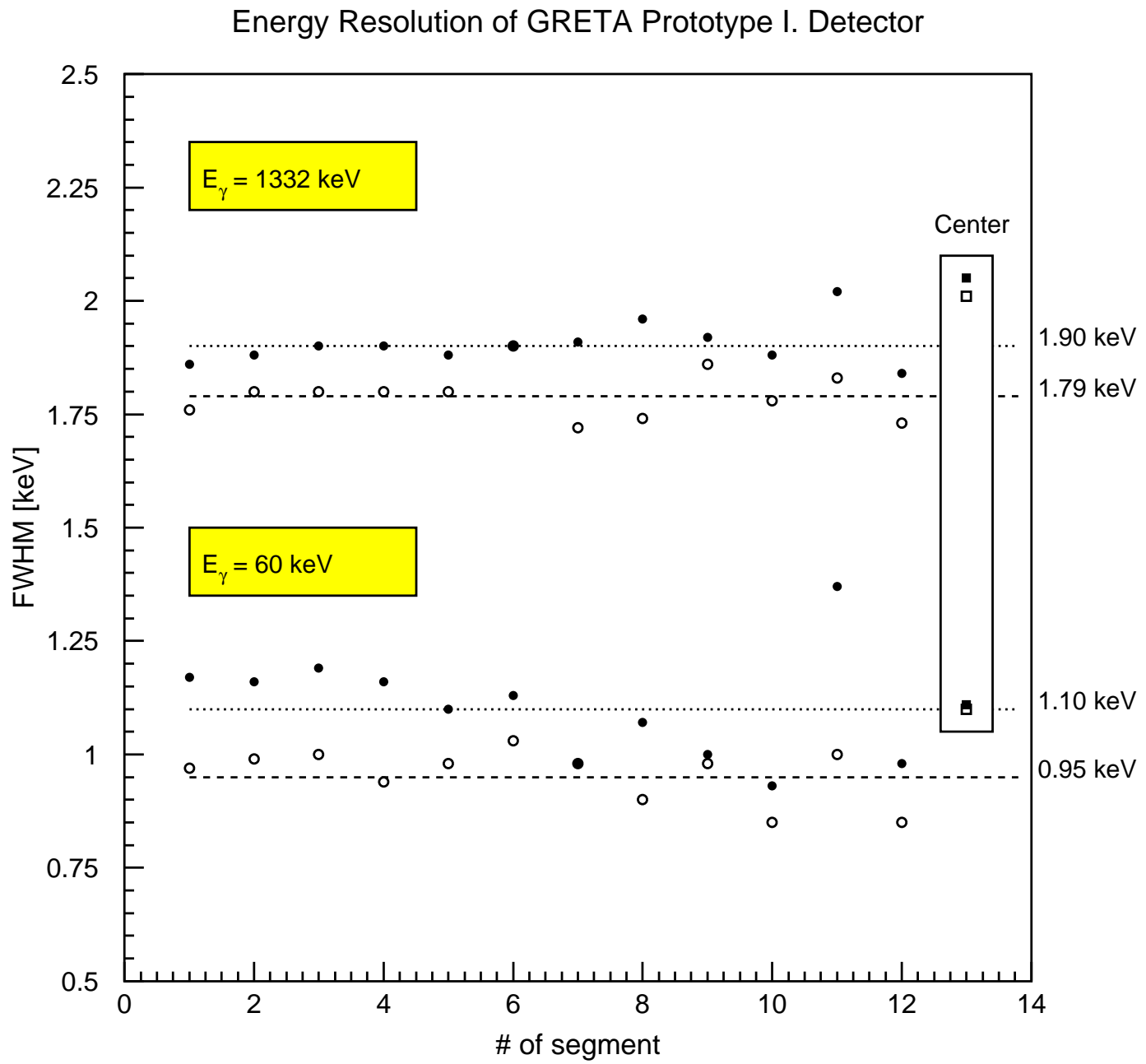

Fig. 2. Measured energy resolution for all 12 segments and the central channel of the first GRETA prototype detector for energies of $60 \mathrm{keV}$ and $1332 \mathrm{keV}$. The open circles show the values provided by the manufacturer while the values measured at LBNL are plotted as solid circles. On the right hand side average segment resolutions are shown. 


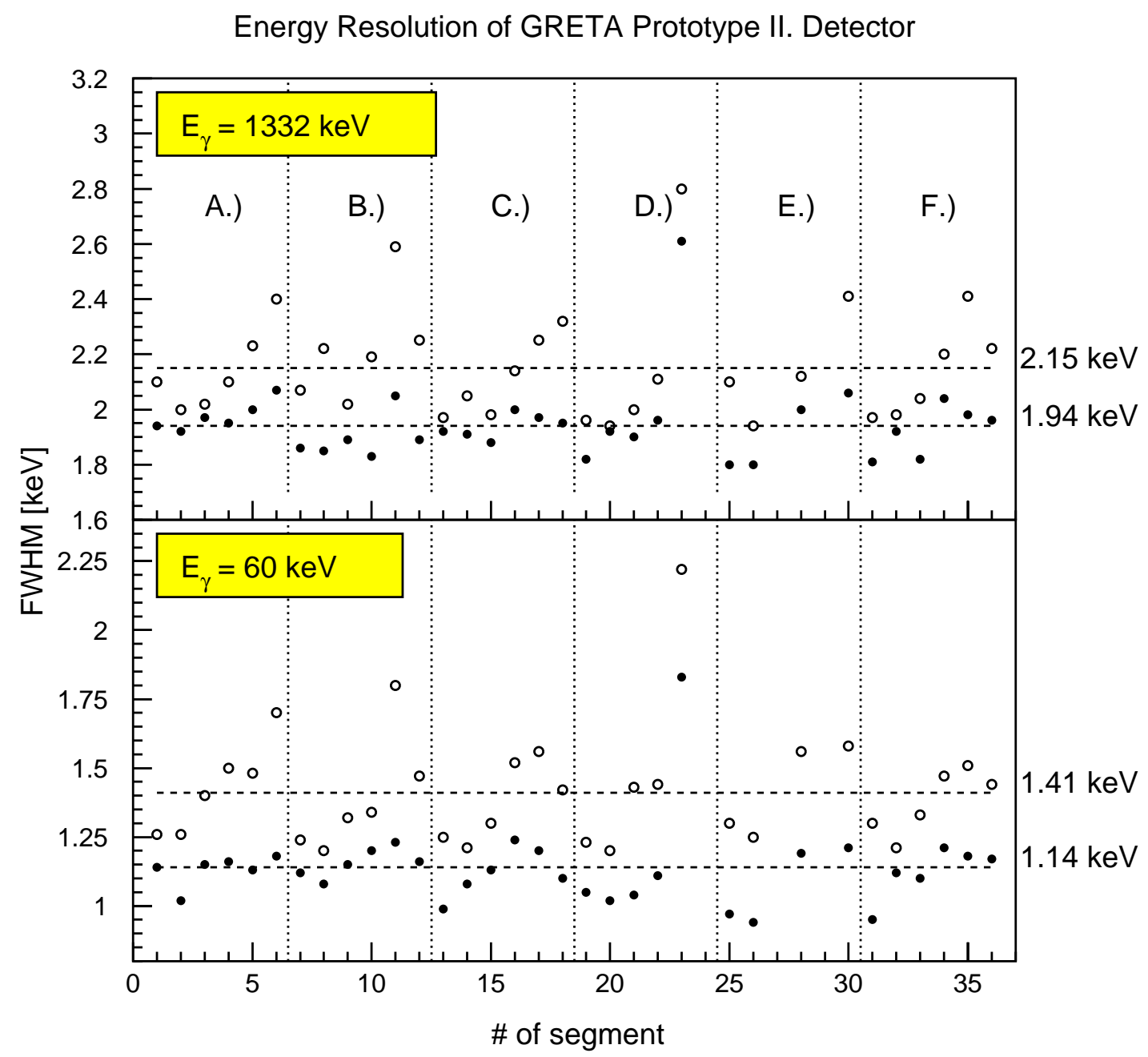

Fig. 3. Measured energy resolution for all working segments of the second GRETA prototype detector for energies of $60 \mathrm{keV}$ and $1332 \mathrm{keV}$. The open circles show the values obtained after all channels have been powered for one day and reached thermal equilibrium at a higher temperature. The solid circles were obtained in a "cold" environment for the FET's. The dashed lines indicate the average values of all segments 

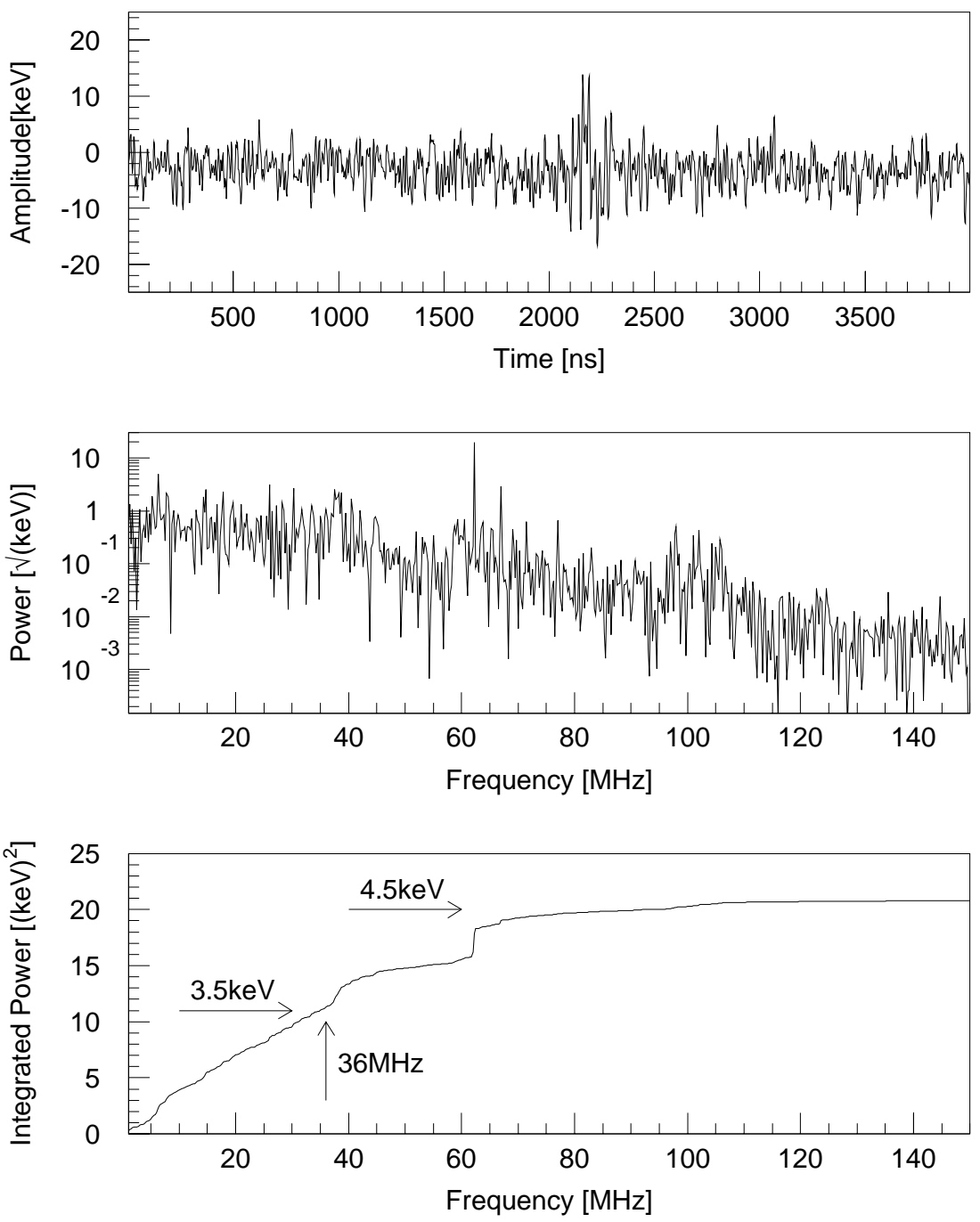

Fig. 4. Measured noise spectrum (top), its power spectrum (middle) and the integrated power spectrum (bottom) from one of the second GRETA prototype segments. 


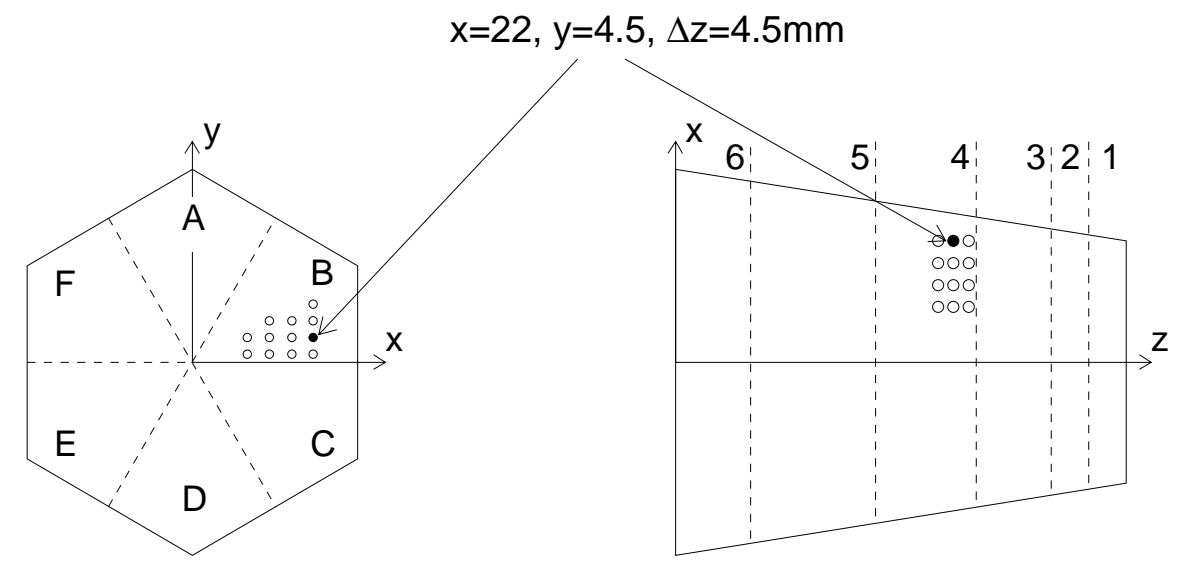

Fig. 5. Coordinate system used for the position sensitivity measurement. The segments are labeled 1-6 in longitudinal direction and A-F in azimuthal direction. Circles indicate $\gamma$-ray interaction points in segment B4 where the measurements were carried out. 


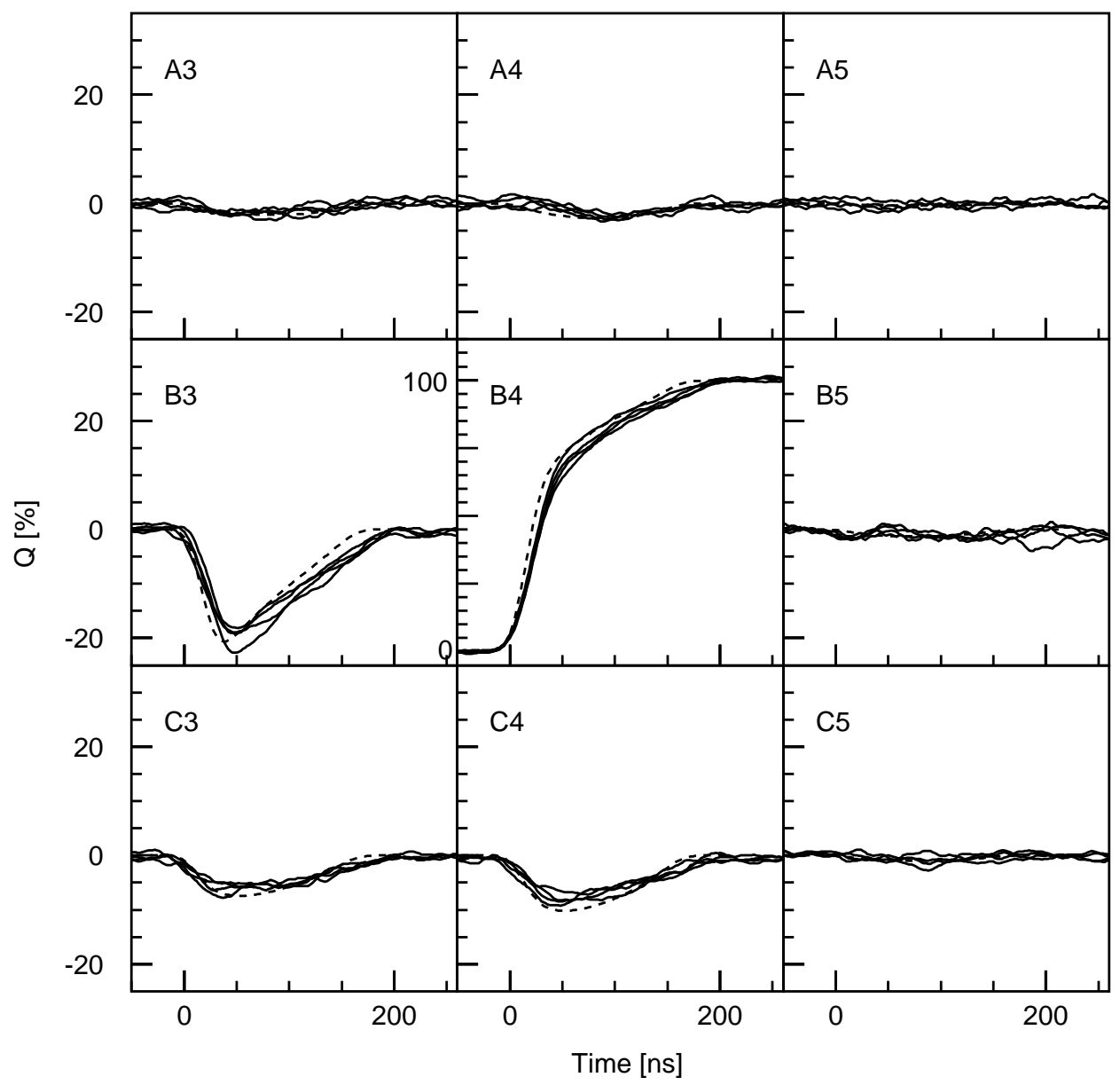

Fig. 6. Measured set of segment signals at $\mathrm{x}=22 \mathrm{~mm}, \mathrm{y}=4.5 \mathrm{~mm}$ and $\mathrm{z}=58.5 \mathrm{~mm}$ $(\Delta \mathrm{z}=1.5 \mathrm{~mm})$. Segment B4 contains the interaction which is $4.5 \mathrm{~mm}$ away from segment $\mathrm{C} 4$ and $1.5 \mathrm{~mm}$ away from segment B3. The dashed lines indicate the calculated signals for this location. 

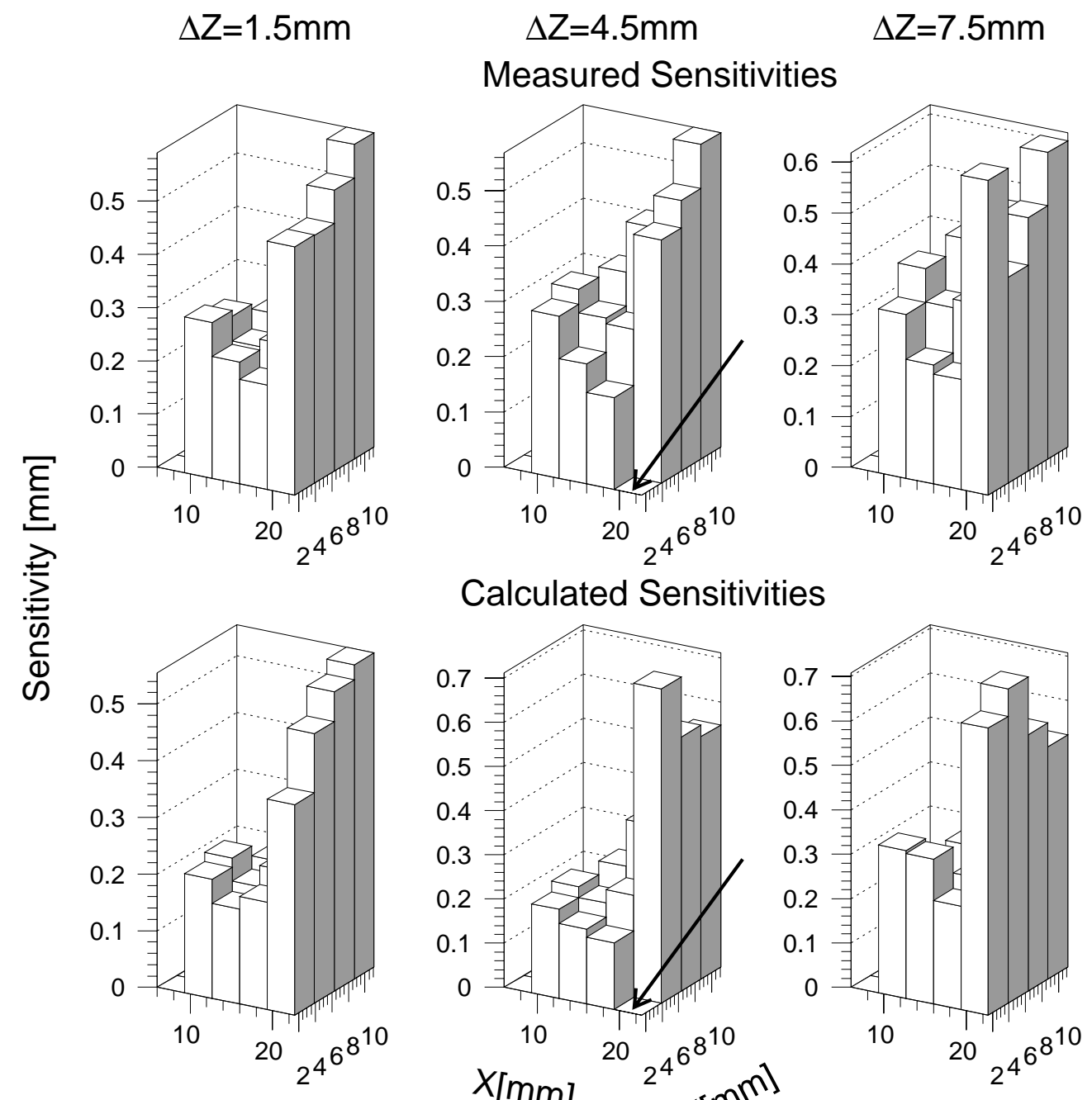

\section{Calculated Sensitivities}
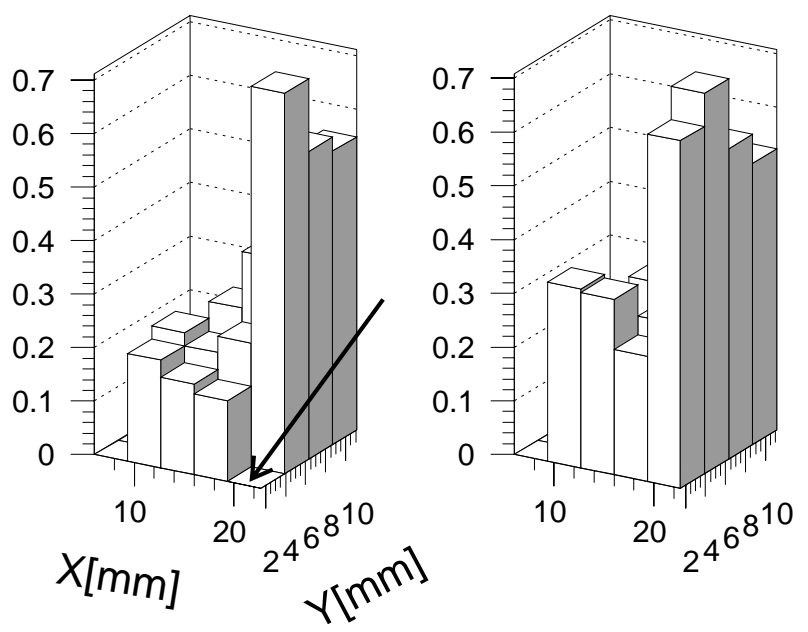

Fig. 7. Sensitivities obtained for the location at $\mathrm{x}=22 \mathrm{~mm}, \mathrm{y}=1.5 \mathrm{~mm}$ and $\mathrm{z}=55.5 \mathrm{~mm}(\Delta \mathrm{z}=4.5 \mathrm{~mm})$, marked by the arrow. 


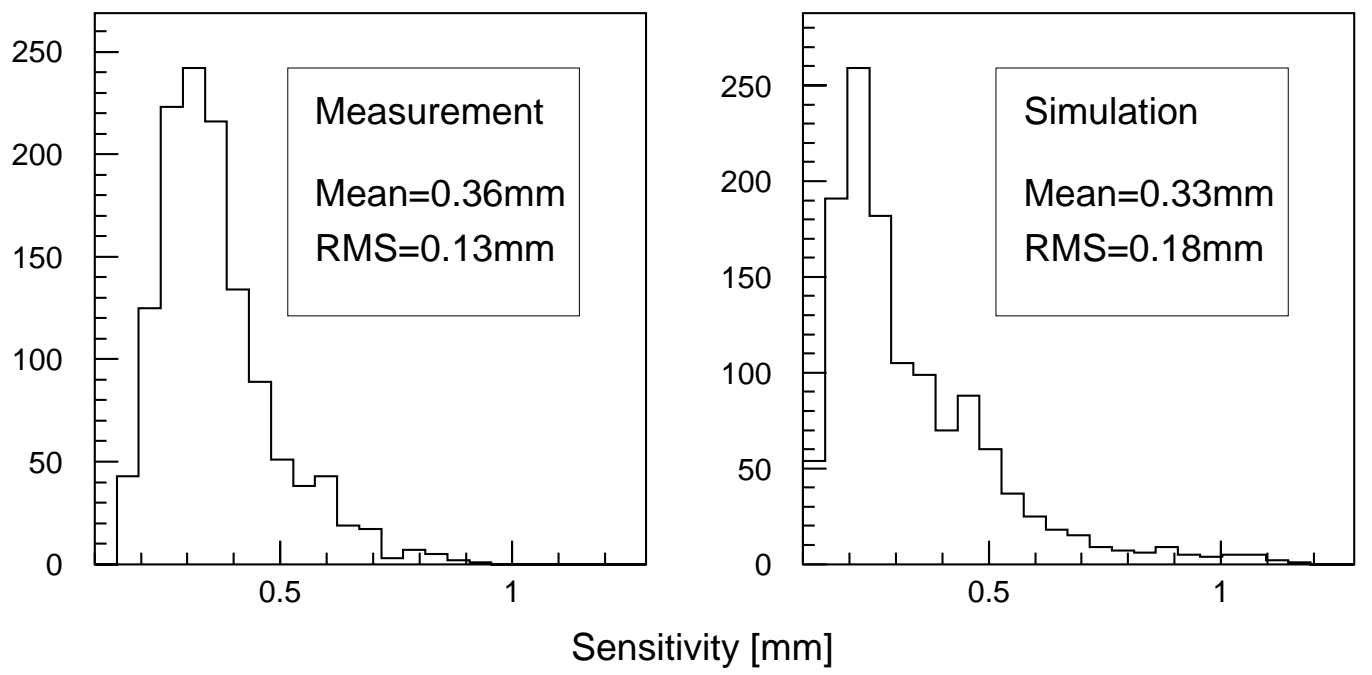

Fig. 8. Sensitivity distributions of all combinations of measured (left) and calculated (right) sensitivities. 


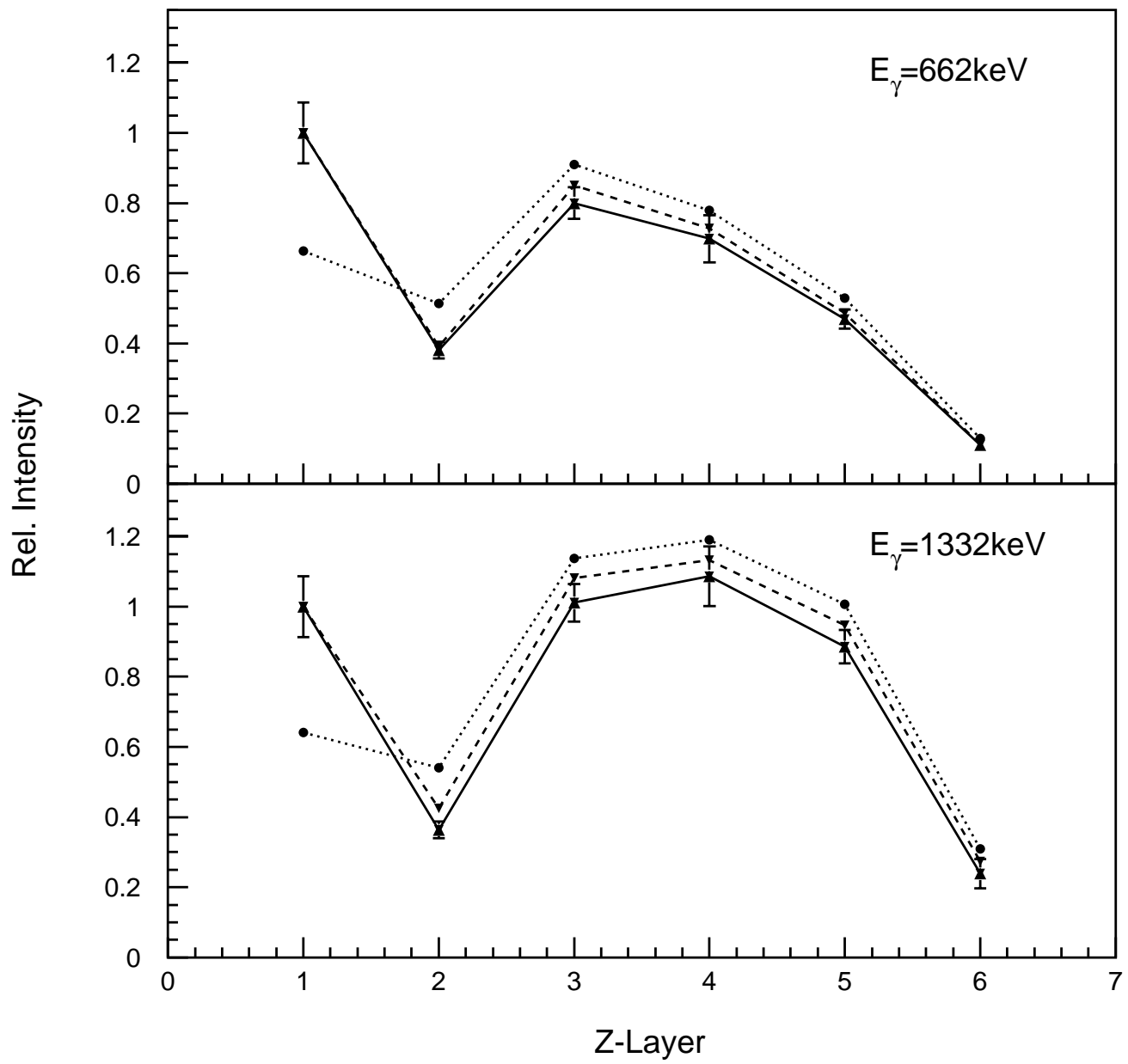

Fig. 9. Relative efficiencies obtained in the 36-fold segmented GRETA prototype detector for B segments in the 6 different depth layers. The solid line represents measured values, the dotted lines shows results of simulations only taking the dimensions of the segments into account and the dashed line shows results of calculations based on the same simulations but following the pathway to the collecting electrode. 


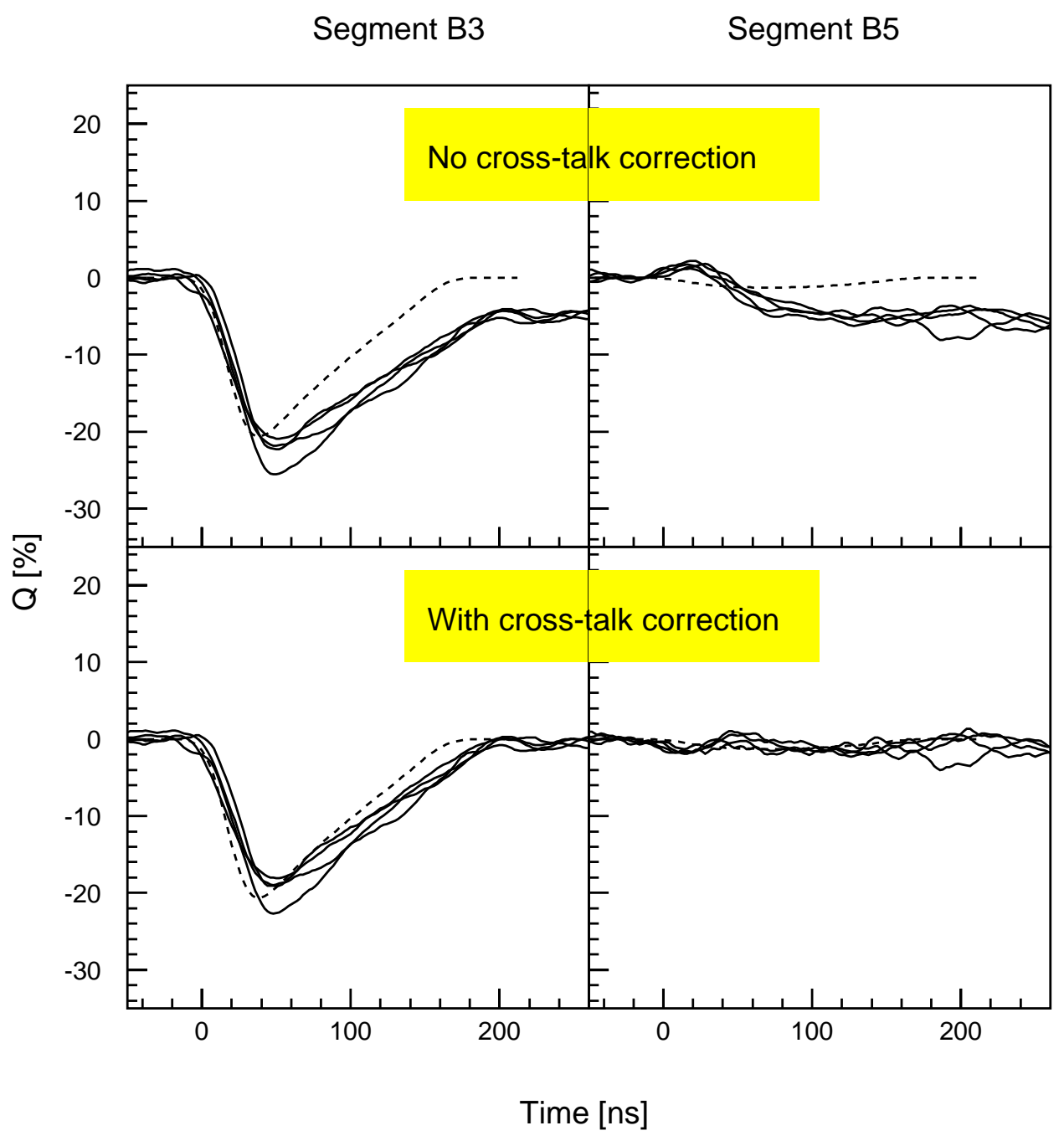

Fig. 10. Signals of segments B3 and B5 as in fig. 6 before (top) and after (bottom) correcting for cross-talk. 